\title{
CRITICAL PHENOMENA AND PHASE TRANSITIONS IN LARGE LATTICES WITHIN MONTE-CARLO BASED NON-PERTURBATIVE APPROACHES
}

\author{
J. KAUPUŽS, ${ }^{1,2}$ J. RIMŠĀNS, ${ }^{1,2}$ R.V.N. MELNIK ${ }^{3}$ \\ ${ }^{1}$ Institute of Mathematics and Computer Science, University of Latvia \\ (29, Rainga Blvd., LV-1459 Riga, Latvia ; e-mail: kaupuzs@latnet. lv) \\ ${ }^{2}$ Institute of Mathematical Sciences and Information Technologies, University of Liepaja \\ (14, Liela Str., Liepaja LV-3401, Latvia) \\ ${ }^{3}$ M2NeT Laboratory, Wilfrid Laurier University \\ (75, University Ave. W, Waterloo, ON, Canada, N2L 3C5; e-mail: rmelnik@wlu. ca)
}

Critical phenomena and Goldstone mode effects in spin models with the $O(n)$ rotational symmetry are considered. Starting with Goldstone mode singularities in the $X Y$ and $O(4)$ models, we briefly review various theoretical concepts, as well as state-of-theart Monte Carlo simulation results. They support recent results of the GFD (grouping of Feynman diagrams) theory, stating that these singularities are described by certain nontrivial exponents, which differ from those predicted earlier by perturbative treatments. Furthermore, we present the recent Monte Carlo simulation results of the three-dimensional Ising model for lattices with linear sizes up to $L=1536$, which are very large as compared to $L \leq 128$ usually used in the finite-size scaling analysis. These results are obtained, using a parallel OpenMP implementation of the Wolff single-cluster algorithm. The finite-size scaling analysis of the critical exponent $\eta$, assuming the usually accepted correctionto-scaling exponent $\omega \approx 0.8$, shows that $\eta$ is likely to be somewhat larger than the value $0.0335 \pm 0.0025$ of the perturbative renormalization group (RG) theory. Moreover, we have found that the actual data can be well described by different critical exponents: $\eta=\omega=1 / 8$ and $\nu=2 / 3$, found within the GFD theory.

\section{Introduction}

Critical phenomena are observed in a vicinity of phase transition points in a variety of systems (e.g., solids - ferromagnets, ferroelectrics; fluids - superfluid $\lambda$ transition; liquid-gas transition, etc.), which manifest themselves in power-like singularities of physical observables described by critical exponents. In many systems, like isotropic ferromagnets (e.g., polycrystalline Ni), a certain singularity is observed not only at the critical temperature $T=T_{c}$, but also at the vanishing external field $h \rightarrow 0$ for any temperature $T<T_{c}$. This phenomenon known as the Goldstone mode singularity is also described by power-like divergences and certain exponents. In this sense, it can be viewed as a critical phenomenon, which takes place in a vicinity of the critical line $h=0$ in the $T-h$ plane.

The vicinity of a critical point (or line) is not the natural domain of validity of any perturbation theory. Therefore, one should resort to the non-perturbative methods such as

- Exact and rigorous analytic solution methods (transfer matrix methods, combinatorial methods, Bethe-ansatz),

- Conformal field theory analysis,

- Non-perturbative renormalization group (RG) analysis,

- Numerical transfer-matrix calculations,

- Monte Carlo (MC) simulations.

The exact solutions [1-3] and the conformal field analysis [4] are powerful tools to determine the critical exponents of two-dimensional models. However, these approaches are not helpful in most of the three-dimensional cases. The MC method can be used here. The MC-based approaches have been successful in a wide range of applications in classical and quantum mechanics, physics, chemistry, biology, astrophysics, nanoscience, heat transport, fluid dynamics, materials science, economics, and finances (e.g., [5-10] and references therein). Today, we see a growing interest in the application of MC-based non-perturbative approaches and the development of powerful MC simulation tools for 3D systems [11, 12]. The exact non-perturbative RG equations are known for various models on fractal lattices (see, e.g., [13] and references therein). Moreover, a rigorous RG analysis has been made in four dimensions (see, e.g., [14]). In most of other cases, the non-perturbative RG equations provide 
only approximations, the accuracy of which cannot be well controlled (see, e.g., [15-17]). To the contrary, the MC method allows one, in principle, to reach any desired accuracy. An advantage of the MC method is in its ability to perform smart moves that can be tuned for each physical system or phenomenon. In particular, the Wolff cluster moves used in this study allow us to overcome the problem of critical slowing down near the phase transition point. It is extremely important in studying the critical phenomena. Therefore, the MC method has no real alternative in a non-perturbative determination of critical exponents of the three-dimensional systems like lattice spin $(O(n))$ models.

The general framework of perturbative methods includes the study of critical point phenomena at $T \rightarrow T_{c}$, as well as the Goldstone mode effects at $T<T_{c}$. Our aim is to verify nonperturbatively the validity of various theoretical perturbative approaches. Hence, the MC test of the Goldstone mode singularities is one of the important points here. We start with the Goldstone mode effects, since this case is simpler in the sense that it does not require the determination of the critical temperature $T_{c}$, i.e., there are less ambiguities as compared to the $T \rightarrow T_{c}$ case.

\section{Goldstone Mode Singularities in the $O(n)$ Models}

Here, we consider such lattice spin models, where the spin is an $n$-component vector with $n \geq 2$. These are called $O(n)$ models due to the $O(n)$ global rotational symmetry exhibited by the $n$-vector model in the absence of an external field. The Hamiltonian $\mathcal{H}$ of such a model reads

$$
\frac{\mathcal{H}}{T}=-\beta\left(\sum_{\langle i j\rangle} \mathbf{s}_{i} \mathbf{s}_{j}+\sum_{i} \mathbf{h} \mathbf{s}_{i}\right)
$$

where $T$ is the temperature measured in energy units, $\mathbf{s}_{i}$ is the spin variable ( $n$-component vector of unit length) of the $i$-th lattice site, $\beta$ is the coupling constant, and $\mathbf{h}$ is the external field with magnitude $|\mathbf{h}|=h$. The summation takes place over all pairs $\langle i j\rangle$ of the nearest neighbors in the lattice.

In the thermodynamic limit $L \rightarrow \infty$ below the critical point, i.e., at $\beta>\beta_{c}$, the magnetization $M(h)$ and the Fourier-transformed transverse $\left(G_{\perp}(\mathbf{k})\right)$ and longitudinal $\left(G_{\|}(\mathbf{k})\right)$ two-point correlation functions exhibit Goldstone mode power-law singularities:

$$
M(h)-M(+0) \propto h^{\rho} \quad \text { at } \quad h \rightarrow 0
$$

$$
\begin{aligned}
& G_{\perp}(\mathbf{k}) \propto k^{-\lambda_{\perp}} \quad \text { at } h=+0 \quad \text { and } \quad k \rightarrow 0, \\
& G_{\|}(\mathbf{k}) \propto k^{-\lambda_{\|}} \quad \text { at } h=+0 \text { and } k \rightarrow 0 .
\end{aligned}
$$

According to the standard theory, including the perturbative RG method [18-21], as well as the effective Lagrangians approach [22], $\lambda_{\perp}=2$ and $\lambda_{\|}=4-d$ hold for $2<d<4$, and $\rho=1 / 2$ is true in three dimensions. More nontrivial universal values are expected according to [23], such that

$d / 2<\lambda_{\perp}<2$

$\lambda_{\|}=2 \lambda_{\perp}-d$

$\rho=\left(d / \lambda_{\perp}\right)-1$

hold for $2<d<4$. These relations have been obtained in [23] by analyzing self-consistent diagram equations for the correlation functions without cutting the perturbation series. This approach is based on a certain grouping of Feynman diagrams introduced in [24], and therefore is called the GFD theory.

MC simulations have been performed [25-27] to test these relations for the $O(2)$ and $O(4)$ models on a simple cubic lattice. The estimate $\lambda_{\perp}=1.955 \pm 0.020$ has been found in [27] for the transverse correlation function, where it has been stated also that the behavior of the longitudinal correlation function is well consistent with $\lambda_{\|} \approx 0.9$. According to $(7)$, we have $1 / 2<\rho<1$ in three dimensions. A reasonable numerical evidence for this relation has been obtained in [26] from the susceptibility data, where the MC estimate $\rho=0.555(17)$ has been reported for the $3 \mathrm{D} X Y(O(2))$ model. It corresponds to $\lambda_{\perp}=1.929(21)$ according to $(7)$.

\section{Critical Point Singularities in the $n$-Vector Models}

In a vicinity of the phase transition point, various quantities have often power-law singularities, which are described by the critical exponents. For three-dimensional systems, exact results are difficult to obtain, and one usually relies on approximate methods. A review of standard perturbative RG methods, applied here, can be found, e.g., in [28-32]. The results of the Borelresummation of the perturbation series for the critical exponents obtained within this approach are reported in [33]. We will further focus on the Monte Carlo testing of the theoretical predictions for the 3D Ising 
model, which is a particular case of $n=1$. The critical exponent $\eta$, describing the $\sim k^{-2+\eta}$ singularity of the critical correlation function $G^{*}(\mathbf{k})$, is of particular interest here. According to [33], the most accurate theoretical value $\eta=0.0335 \pm 0.0025$ is obtained from the series at the fixed dimension $d=3$. The results of the resummation of the $\epsilon$-expansion in [33] are $\eta=0.036 \pm 0.005$ and $\eta=0.0365 \pm 0.0050$. If all these estimates are correct within the error bars, then we have $0.0315<\eta<0.036$. This estimation fairly well agrees with the value $\eta=0.0366(8)$ extracted from the finitesize scaling analysis of the MC data within $L \in[10,128]$, reported in [34]. We will present the $\mathrm{MC}$ results for much larger lattices up to $L=1536$ to test the agreement more precisely. The other exponents relevant here are the correlation length exponent $\nu$ and the correction-toscaling exponent $\omega$. The widely accepted values for the $3 \mathrm{D}$ Ising model are $\nu \simeq 0.63$ and $\omega \simeq 0.8$. They are in agreement, e.g., with the estimates $\nu=0.6304 \pm 0.0013$ and $\omega=0.799 \pm 0.011$ reported in [33].

As discussed in Sec. 2, the alternative theoretical approach, called the GFD theory, provides promising results concerning the Goldstone mode singularities. Therefore, it is interesting to verify the predictions of this theory also for the critical point singularities. In [24], a set of possible values of the exact critical exponents for the $\varphi^{4}$ model in two $(d=2)$ and three $(d=3)$ dimensions has been proposed:

$\gamma=\frac{d+2 j+4 i}{d(1+i+j)-2 j} ; \quad \nu=\frac{2(1+i)+j}{d(1+i+j)-2 j}$,

where $\gamma$ is the susceptibility exponent, $i$ may have a natural value starting with 1 , and $j$ is an integer equal or larger than $-i$. Other critical exponents can be calculated from these ones, using the known scaling relations. These values agree with the known exact solutions of the two-dimensional Ising model $(i=3, j=0)$ and of the spherical model $(j / i \rightarrow \infty)$. A prediction has been made also for the $3 \mathrm{D}$ Ising model, i.e., $\gamma=5 / 4$ and $\nu=2 / 3$, corresponding to $i=3$ and $j=0$, as in the two-dimensional case. This value of $\nu$ is consistent with the logarithmic singularity of specific heat (according to $\alpha=2-d \nu=0$ ) proposed earlier by Tseskis [35]. The exponents $\gamma=5 / 4$ and $\nu=2 / 3$ have been later conjectured for the 3D Ising model by Zhang [36]. The critical exponents $\gamma=9 / 8$ and $\nu=5 / 8$, calculated for the liquid-gas system by Bondarev $[37,38]$, also correspond to (8). In this case, $i=2$ and $j=-1$ hold. As explained in [24], Eqs. (8) are meaningful for a positive integer $n$, and we can have $j=j(n)$ and $i=i(n)$ in the case where the order parameter is an $n$-component vector. The spatial dimensionality $d$ might be considered as a continuous parameter in (8) within $2 \leq d \leq 4$. At $n=1$, the second-order phase transition at a finite temperature can be expected also for $d<2$. However, according to the discussion in [39], an analytic extension of (8) to this region, probably, is only formal and does not correspond to any real system (fractal lattice).

According to [24], corrections to scaling can be represented by an expansion of the correction factor (amplitude) in integer powers of $t^{2 \nu-\gamma}$ and $t^{2 \gamma-d \nu}$ at $t \rightarrow 0$, where $t$ is the reduced temperature. This expansion is simplified, since $(2 \gamma-d \nu) /(2 \nu-\gamma)$ is an integer number according to (8), and $2-\gamma / \nu=\eta$ holds according to the known scaling relation. Hence, we obtain the expansion in powers of $t^{\theta}$, where $\theta=\eta \nu$. In other words, the correction-to-scaling exponent in the expansions at $t \rightarrow 0$ is $\theta=\eta \nu$, and the corresponding correctionto-scaling exponent in the finite-size-scaling analysis is $\omega=\theta / \nu=\eta$, if the first expansion coefficient is nonzero. Allowing that some of the expansion coefficients are zero, we can have $\theta=\ell \eta \nu$, where $\ell$ is a positive integer. However, our actual MC data support the most natural choice of $\ell=1$ for the 3D Ising model. This implies that the expansion coefficients do not vanish in general, but the nontrivial correction terms vanish only in some special cases, as the 2D Ising model or the spherical model, where $\omega=1$ and/or $\theta=1$. According to the numerical transfer matrix calculations in [40], a nontrivial correction to finite-size scaling with the exponent $\omega=\eta$, probably, exists in the two-point correlation function even in the $2 \mathrm{D}$ Ising model, although its amplitude is very small here.

The discussed GFD critical exponents $\eta=1 / 8$ and $\nu=2 / 3$ for $n=1$ seem to be quite incompatible with the MC data of the 3D Ising model, if we assume the usual correction-to-scaling exponent $\omega \simeq 0.8$. However, it turns out that our data for large enough sizes are very well consistent even with the set of exponents $\eta=\omega=$ $1 / 8$ and $\nu=2 / 3$. The disagreement of these exponents with those of the perturbative RG method can also be understood based on a critical analysis [41].

\section{Monte Carlo Simulation Results for the 3D Ising Model}

We have simulated the 3D Ising model on a simple cubic lattice (at $h=0$ ), using the iterative method introduced in [42] to adjust the coupling $\beta$ to the pseudocritical coupling $\widetilde{\beta}_{c}$, which corresponds to certain value 1.6 of the quantity $U=\left\langle m^{4}\right\rangle /\left\langle m^{2}\right\rangle^{2}$, where $m$ is the magne- 
tization per spin. The pseudocritical coupling $\widetilde{\beta}_{c}$ tends to the true critical coupling $\beta_{c}$ as $L \rightarrow \infty$. By this method, we estimate the moments of energy $\varepsilon$ and magnetization $m$ per spin, i.e., $\left\langle\varepsilon^{k} m^{l}\right\rangle$ for a set of $\beta$ values fluctuating around $\widetilde{\beta}_{c}$, and then calculate these mean values and related quantities at any given $\beta$ near $\widetilde{\beta}_{c}$ by using the Taylor series expansion for $\ln \left\langle(-\varepsilon)^{k} m^{l}\right\rangle$. We have evaluated $\widetilde{\beta}_{c}(L)$ for various lattice sizes $L$ and then estimated $\beta_{c}$ by fitting these $\widetilde{\beta}_{c}(L)$ data. In addition, we have calculated the susceptibility $\chi=L^{3}\left\langle m^{2}\right\rangle$ and the derivative $\partial Q / \partial \beta$, where $Q=1 / U$, at $\beta=\widetilde{\beta}_{c}(L)$, as well as at certain estimated critical couplings $\beta_{c}$, in order to make a finite-size scaling analysis of the critical exponents.

This method has been tested and discussed in detail in [42], giving also some data for $L \leq 1024$. Here, we have extended the simulation results up to $L=1536$. A parallel (OpenMP) implementation of the Wolff single cluster algorithm has been used in our simulations, combining it with a sophisticated shuffling scheme for the generation of a high-quality pseudorandom numbers, as described in [42]. Several tests have been made in [42] to verify the quality of the pseudorandom numbers. Here, we have tested and used also the lagged Fibonacci pseudorandom number generator (PRNG) with multiplication operation and the lags $r=24, s=55$ (see [10]). We have improved it with the standard shuffling scheme [10], using the shuffling box of the size 10000 in this case. Although the lagged Fibonacci generator with additive operation produces certain correlations in the sequence of pseudorandom numbers, which can be well detected by the directed-random-walk test [43], this defect is practically not observed in the actual multiplicative case. Like in [42], we have verified it by performing such test with $10^{12}$ trajectories. Good results have been obtained in this test both for the original PRNG and for the one improved by the shuffling. It indicates that the multiplicative lagged Fibonacci generator works well in applications with cluster algorithms, as it also has been mentioned in [44].

The simulations of the two largest lattices with $L=$ 1280 and $L=1536$ have been performed with the shuffling scheme of [42] and partly (24 usable iterations from 56 or 54 ones at $L=1280$ and $L=1536$, respectively) also with the improved lagged Fibonacci generator. For complete confidence, we have verified that the simulation results of the two generators agree within the statistical error bars, whereas the final simulated values have been obtained by summarizing the data from both of them. Note that one iteration included 5280000 MC (Wolff cluster algorithm) steps at $L=1280$ and $6720000 \mathrm{MC}$ steps at $L=1536$, the MC measurements being performed after each 160 and 192 steps, respectively.

Our simulation results for the pseudocritical coupling $\widetilde{\beta}_{c}$, as well as for the corresponding values of $\chi / L^{2}$ and $\partial Q / \partial \beta$ depending on the lattice size $L$ are given in Table 1. In Table 2, we give the latter two quantities calculated at $\beta=0.221654604$ and $\beta=0.221654615$, corresponding to two different estimates of the critical coupling. In addition, we have calculated these quantities also at $\beta=0.2216545$ for $L \leq 128$ and have verified that the values agree within the error bars with those reported for this $\beta$ in [34].

\section{Estimation of the Critical Coupling}

We have evaluated the critical coupling $\beta_{c}$ by fitting the $\widetilde{\beta}_{c}(L)$ data to the finite-size scaling ansatz

$\widetilde{\beta}_{c}(L) \simeq \beta_{c}+L^{-1 / \nu}\left(a_{0}+a_{1} L^{-\omega}+a_{2} L^{-2 \omega}\right)$,

T a b l e 1. The values of $\widetilde{\beta}_{c}$, as well as $\chi / L^{2}$ and $10^{-3} \partial Q / \partial \beta$ at $\beta=\widetilde{\beta}_{c}$ depending on $L$

\begin{tabular}{c|c|c|c}
\hline$L$ & $\widetilde{\beta}_{c}$ & $\chi / L^{2}$ & $10^{-3} \partial Q / \partial \beta$ \\
\hline 1536 & $0.2216546081(114)$ & $1.1900(22)$ & $96.01(73)$ \\
1280 & $0.2216546524(136)$ & $1.2009(25)$ & $72.04(53)$ \\
1024 & $0.221654625(22)$ & $1.2046(28)$ & $50.57(45)$ \\
864 & $0.221654635(25)$ & $1.2165(21)$ & $38.71(25)$ \\
768 & $0.221654672(27)$ & $1.2212(20)$ & $31.89(20)$ \\
640 & $0.221654615(31)$ & $1.2281(17)$ & $23.95(12)$ \\
512 & $0.221654662(45)$ & $1.2367(16)$ & $16.785(77)$ \\
432 & $0.221654637(58)$ & $1.2450(18)$ & $12.907(59)$ \\
384 & $0.221654567(65)$ & $1.2480(16)$ & $10.627(50)$ \\
320 & $0.221654716(75)$ & $1.2578(16)$ & $7.967(36)$ \\
256 & $0.22165460(11)$ & $1.2656(15)$ & $5.577(24)$ \\
216 & $0.22165460(13)$ & $1.2726(12)$ & $4.288(14)$ \\
192 & $0.22165425(16)$ & $1.2734(14)$ & $3.533(14)$ \\
160 & $0.22165414(18)$ & $1.2818(11)$ & $2.6495(87)$ \\
128 & $0.22165430(20)$ & $1.2913(10)$ & $1.8643(49)$ \\
108 & $0.22165376(26)$ & $1.2969(10)$ & $1.4170(36)$ \\
96 & $0.22165369(32)$ & $1.3012(10)$ & $1.1796(28)$ \\
80 & $0.22165278(32)$ & $1.30659(74)$ & $0.8822(18)$ \\
64 & $0.22165159(52)$ & $1.31466(78)$ & $0.6192(11)$ \\
54 & $0.22164968(56)$ & $1.31916(76)$ & $0.47334(81)$ \\
48 & $0.22164790(69)$ & $1.32164(66)$ & $0.39331(63)$ \\
40 & $0.22164383(80)$ & $1.32562(64)$ & $0.29424(40)$ \\
32 & $0.22163444(98)$ & $1.32835(59)$ & $0.20586(25)$ \\
27 & $0.2216212(11)$ & $1.32829(52)$ & $0.15703(16)$ \\
24 & $0.2216125(12)$ & $1.33027(47)$ & $0.13076(13)$ \\
20 & $0.2215821(17)$ & $1.32860(42)$ & $0.097717(87)$ \\
16 & $0.2215235(18)$ & $1.32510(34)$ & $0.068538(48)$ \\
\hline & & &
\end{tabular}




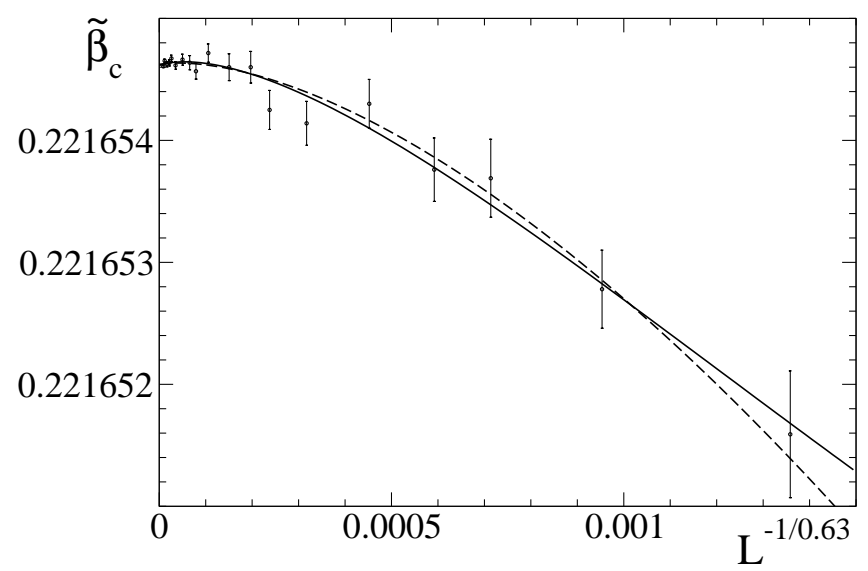

Fig. 1. The pseudocritical coupling $\widetilde{\beta}_{c}$ vs $L^{-1 / 0.63}$. The dashed line shows the fit to (9), including only the leading correction term to $\widetilde{\beta}_{c}-\beta_{c}$ with coefficient $a_{1}$ and exponents $\nu=0.63$ and $\omega=0.8$, whereas the solid line is the fit with both correction terms included

T a b l e 2. The values of $\chi / L^{2}$ and $10^{-3} \partial Q / \partial \beta$ at $\beta=0.221654604$ and $\beta=0.221654615$ depending on $L$

\begin{tabular}{c|c|c|c|c}
\hline \multirow{2}{*}{$L$} & \multicolumn{2}{|c|}{$\beta=0.221654604$} & \multicolumn{2}{c}{$\beta=0.221654615$} \\
\cline { 2 - 5 } & $\chi / L^{2}$ & $10^{-3} \partial Q / \partial \beta$ & $\chi / L^{2}$ & $10^{-3} \partial Q / \partial \beta$ \\
\hline 1536 & $1.1882(38)$ & $95.94(69)$ & $1.1930(38)$ & $96.12(69)$ \\
1280 & $1.1849(30)$ & $71.62(45)$ & $1.1885(30)$ & $71.72(45)$ \\
1024 & $1.1998(37)$ & $50.48(40)$ & $1.2023(37)$ & $50.53(40)$ \\
864 & $1.2110(32)$ & $38.63(22)$ & $1.2130(32)$ & $38.66(22)$ \\
768 & $1.2110(31)$ & $31.77(18)$ & $1.2126(32)$ & $31.79(18)$ \\
640 & $1.2268(27)$ & $23.94(10)$ & $1.2280(27)$ & $23.95(10)$ \\
512 & $1.2321(27)$ & $16.756(69)$ & $1.2330(27)$ & $16.762(69)$ \\
432 & $1.2429(24)$ & $12.898(52)$ & $1.2436(24)$ & $12.901(52)$ \\
384 & $1.2499(24)$ & $10.634(44)$ & $1.2505(24)$ & $10.636(44)$ \\
320 & $1.2534(22)$ & $7.955(33)$ & $1.2538(22)$ & $7.956(33)$ \\
256 & $1.2656(23)$ & $5.577(22)$ & $1.2659(23)$ & $5.577(22)$ \\
216 & $1.2727(21)$ & $4.288(13)$ & $1.2729(21)$ & $4.288(13)$ \\
192 & $1.2795(21)$ & $3.540(12)$ & $1.2797(21)$ & $3.541(12)$ \\
160 & $1.2879(17)$ & $2.6550(78)$ & $1.2880(17)$ & $2.6551(78)$ \\
128 & $1.2941(13)$ & $1.8660(42)$ & $1.2942(13)$ & $1.8661(42)$ \\
108 & $1.3028(13)$ & $1.4198(32)$ & $1.3029(13)$ & $1.4198(32)$ \\
96 & $1.3066(14)$ & $1.1817(25)$ & $1.3066(14)$ & $1.1817(25)$ \\
80 & $1.3147(11)$ & $0.8845(16)$ & $1.3147(11)$ & $0.8845(16)$ \\
64 & $1.3241(12)$ & $0.62115(96)$ & $1.3241(12)$ & $0.62116(96)$ \\
54 & $1.33098(85)$ & $0.47518(69)$ & $1.33101(85)$ & $0.47518(69)$ \\
48 & $1.33504(93)$ & $0.39503(54)$ & $1.33506(93)$ & $0.39503(54)$ \\
40 & $1.34177(77)$ & $0.29577(34)$ & $1.34179(77)$ & $0.29577(34)$ \\
32 & $1.34958(71)$ & $0.20728(21)$ & $1.34960(71)$ & $0.20728(21)$ \\
27 & $1.35514(60)$ & $0.15838(14)$ & $1.35515(60)$ & $0.15838(14)$ \\
24 & $1.35836(53)$ & $0.13192(11)$ & $1.35836(53)$ & $0.13192(11)$ \\
20 & $1.36471(61)$ & $0.098821(75)$ & $1.36471(61)$ & $0.098821(75)$ \\
16 & $1.37072(45)$ & $0.069508(41)$ & $1.37072(45)$ & $0.069508(41)$ \\
\hline & & & & \\
& & & & \\
& & &
\end{tabular}

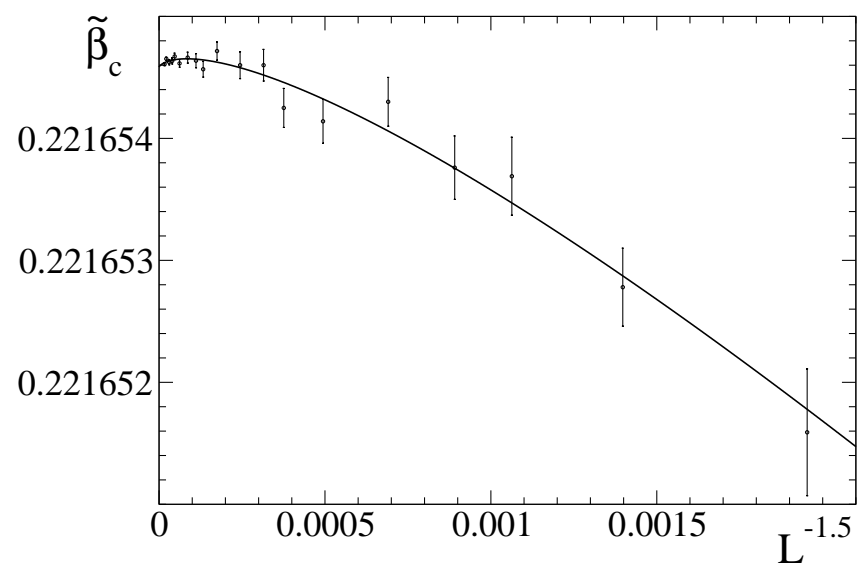

Fig. 2. The pseudocritical coupling $\widetilde{\beta}_{c}$ vs $L^{-3 / 2}$. The solid curve shows the fit to (9), including only the leading correction term to $\widetilde{\beta}_{c}-\beta_{c}$ with coefficient $a_{1}$ and exponents $\nu=2 / 3$ and $\omega=1 / 8$

neglecting higher order corrections. The fits with the usual (RG) exponents $\nu=0.63$ and $\omega=0.8$ within the range $L \in[64,1536]$ are shown in Fig. 1 .

The dashed line shows the fit with only the leading correction term (the term with coefficient $a_{1}$ ) to $\widetilde{\beta}_{c}-\beta_{c}$ included, whereas the solid line - the fit with both correction terms. These fits give $\beta_{c}=0.2216546234(99)$ and $\beta_{c}=0.221654615(13)$, respectively. The $\chi^{2} /$ d.o.f. of both these fits is 1.08 . The data for $L<64$ are omitted here, since inclusion of these relatively small sizes only increases the systematic errors without an essential reduction of the statistical errors.

Surprisingly, the $\widetilde{\beta}_{c}$ data within $L \in[64,1536]$ can be even better fit with the exponents $\nu=2 / 3$ and $\omega=$ $1 / 8$ of the GFD theory. The fit, with only the leading correction to $\widetilde{\beta}_{c}-\beta_{c}$ included, is shown in Fig. 2 . It yields $\beta_{c}=0.221654585(15)$. In this case, $\chi^{2} /$ d.o.f. has the value 0.97 , which is even smaller than for the fits with $\nu=0.63$ and $\omega=0.8$.

Inclusion of the second correction term changes the result only slightly, i.e., it gives $\beta_{c}=0.221654588(47)$ with $\chi^{2} /$ d.o.f. $=1.03$, and the fit curve lies almost on the top of that shown in Fig. 2. The statistical error, however, is strongly increased in this case.

Alternatively, the critical coupling $\beta_{c}$ can be determined from the susceptibility data, requiring the consistency with the finite-size scaling ansatz

$\chi \simeq L^{2-\eta}\left(b_{0}+b_{1} L^{-\omega}+b_{2} L^{-2 \omega}\right)$

or with the corresponding ansatz for the effective exponent

$\eta_{\mathrm{eff}}(L) \simeq \eta+c_{1} L^{-\omega}+c_{2} L^{-2 \omega}$ 


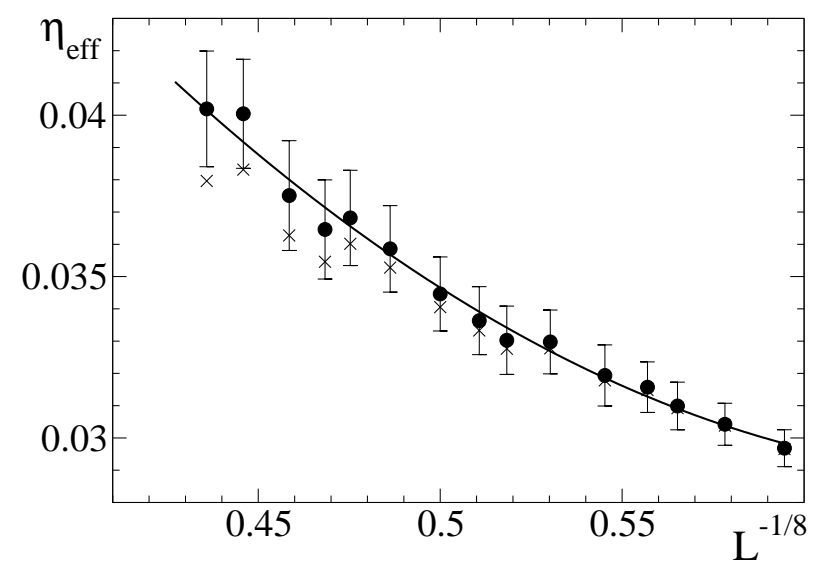

Fig. 3. The effective exponent $\eta_{\text {eff }}$ vs $L^{-1 / 8}$ at $\beta=0.221654604$ (solid circles) and $\beta=0.221654615(\mathrm{x})$. The solid line is the fit of circles to (11) with $\eta=\omega=1 / 8$

at $\beta=\beta_{c}$. Here, the effective exponent $\eta_{\text {eff }}(L)$ is the local slope of the $-\ln \left(\chi / L^{2}\right)$ vs $\ln L$ plot evaluated from the linear fit within $[L / 2,2 L]$. We observe that the plot of the effective exponent within $L \in[64,768]$ (evaluated from the data within $L \in[32,1536]$ ) is optimally described by (11) with $\eta=\omega=1 / 8$ at $\beta \simeq 0.221654604$, as shown in Fig. 3.

From this, we conclude that if the exponents $\eta=\omega=$ $1 / 8$ are correct, then $\beta_{c}=0.221654604(18)$. This is the estimate of $\beta$ at which the fit to (11) with $\omega=1 / 8$ and $\eta$ as a fit parameter yields $\eta=1 / 8$ within the statistical error bars. The solid-line fit at $\beta=0.221654604$ is really good, and the effective exponents can be quite well fit with $\eta=\omega=1 / 8$ also at $\beta=0.221654615$ (the data shown by " $\mathrm{x}$ "), which is the best $\beta_{c}$ value provided by the already considered estimation with the RG exponents.

The value $\beta_{c}=0.221654604(18)$ is our best estimate of the critical coupling in the case where we use the exponents of the GFD theory, since two corrections to scaling are included, giving smaller statistical error bars than those for the other estimate $\beta_{c}=$ $0.221654588(47)$ with two corrections included. Our estimated value $\beta_{c}=0.221654604(18)$ is sufficiently reasonable and plausible, since it agrees well within the error bars with all other estimations considered here, as well as with the most accurate values of $\beta_{c}$ provided by other authors, i.e., $\beta_{c}=0.22165455$ (5) [45] and $\beta_{c}=0.22165457(3)$ [46].

Concerning the standard RG exponents, the method of fitting $\eta_{\text {eff }}$ data is less convenient, since it is sensitive to the precise value of $\eta$, which is not known accurately enough in this approach.

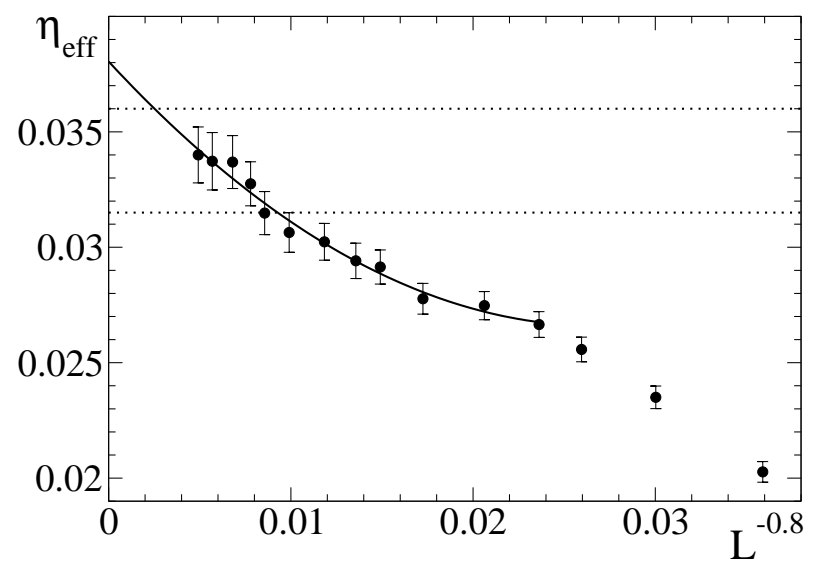

Fig. 4. The effective exponent $\eta_{\text {eff }}$ vs $L^{-0.8}$ at $\beta=\widetilde{\beta}_{c}(L)$. The solid curve is the fit to $(11)$ with fixed $\omega=0.8$. The dotted lines indicate the range $[0.0315,0.036]$ of $\eta$ values consistent with the set of perturbative RG estimations in [33]

\section{Finite-size Scaling Analysis of the Critical Exponents}

\subsection{Tests of the RG exponents}

In order to test the results of the perturbative RG theory, we have estimated the critical exponent $\eta$ by fitting the susceptibility data to (10) and also by fitting the $\eta_{\text {eff }}$ data to (11) at the fixed RG exponent $\omega=0.8$. Note that (10) and (11) are valid at $\beta=\beta_{c}$, as well as at $\beta=\widetilde{\beta}_{c}(L)$. The fits have been made at $\beta=\widetilde{\beta}_{c}(L)$ and at the critical coupling values estimated in Sec. 5 by using the RG exponents.

We start with the fitting of the effective exponent, since this method can be better controlled to see which fits are most appropriate. The plot of $\eta_{\text {eff }}$ vs $L^{-\omega}$ with $\omega=0.8$, evaluated at $\beta=\widetilde{\beta}_{c}(L)$, is shown in Fig. 4 .

We see that the data are well described by a quadratic curve for $L \geq 108$. Therefore, one has to include both correction terms in (11). The fit yields $\eta=0.0380(26)$. This value is slightly larger than the upper limit 0.036 of the interval $0.0315<\eta<0.036$ for the values, which are consistent with the set of perturbative RG estimations of [33] discussed in Sec. 3. However, the discrepancy is within the error bars. Somewhat larger deviations above 0.036 are provided by the fits within $L \geq 64$, shown in Fig. 5, at approximately estimated values $\beta_{c}=0.2216546234(99)$ and $\beta_{c}=0.221654615(13)$ of the critical coupling.

These fits yield $\eta=0.0381(18)$ and $\eta=0.0397(28)$, respectively. The indicated here standard errors are calculated by the jackknife method, taking into ac- 


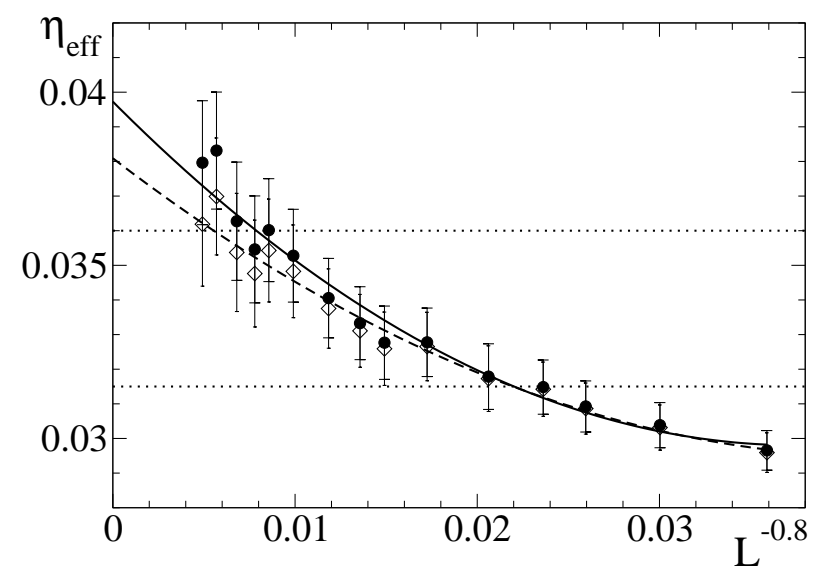

Fig. 5. The effective exponent $\eta_{\text {eff }}$ vs $L^{-0.8}$ at $\beta_{c}=$ 0.2216546234 (99) (diamonds) and $\beta_{c}=0.221654615$ (13) (circles). The curves are the fits to (11) with fixed $\omega=0.8$. The dotted lines indicate the range $[0.0315,0.036]$ of $\eta$ values consistent with the set of perturbative RG estimations in [33]

count the statistical fluctuations in the estimated $\beta_{c}$ value. Namely, the standard error $\sigma$ is evaluated as $\sigma=\sqrt{\sum_{i j} \Delta_{i j}^{2}}$, where $\Delta_{i j}$ is a shift in the estimated $\eta$ value, taking into account also a shift in the fitted $\beta_{c}$ value, when the $j$-th simulation block (iteration) for the $i$-th lattice size is omitted. The statistical correlations are such that the total standard error is smaller than in the case where there are no correlations between the fluctuations in the estimated $\beta_{c}$ value and in the $\eta$ value obtained at a given $\beta$.

We have also evaluated $\eta$ from the fits to (10) at the pseudocritical coupling $\widetilde{\beta}_{c}(L)$, as well as at the two estimates $\beta_{c}=0.2216546234(99)$ and $\beta_{c}=0.221654615(13)$ of the critical coupling. The results of fits within $L \in$ $\left[L_{\min }, 1536\right]$, assuming $\omega=0.8$, are collected in Table 3 .

The best estimates in these three cases are assumed to be $\eta=0.0380(23), \eta=0.0376(20)$ and $\eta=0.0405(25)$, since they perfectly agree with the corresponding esti-

T a b l e 3. The critical exponent $\eta$ evaluated from the fits to (10) within $L \in\left[L_{\min }, 1536\right]$ with fixed $\omega=0.8$. The estimates from the data at $\beta=\widetilde{\beta}_{c}(L)$ and at $\beta=\beta_{c}$ with $\beta_{c}=0.2216546234(99)$ and $\beta_{c}=0.221654615(13)$ are denoted by $\eta_{1}, \eta_{2}$ and $\eta_{3}$, respectively

\begin{tabular}{c|c|c|c}
\hline$L_{\min }$ & $\eta_{1}$ & $\eta_{2}$ & $\eta_{3}$ \\
\hline 32 & $0.0345(12)$ & $0.0363(14)$ & $0.0379(25)$ \\
40 & $0.0353(14)$ & $0.0364(15)$ & $0.0384(27)$ \\
48 & $0.0359(17)$ & $0.0369(17)$ & $0.0394(29)$ \\
54 & $0.0369(19)$ & $0.0376(20)$ & $0.0402(28)$ \\
64 & $0.0380(23)$ & $0.0376(30)$ & $0.0405(25)$ \\
80 & $0.0377(37)$ & $0.0366(38)$ & $0.0398(32)$ \\
\hline
\end{tabular}

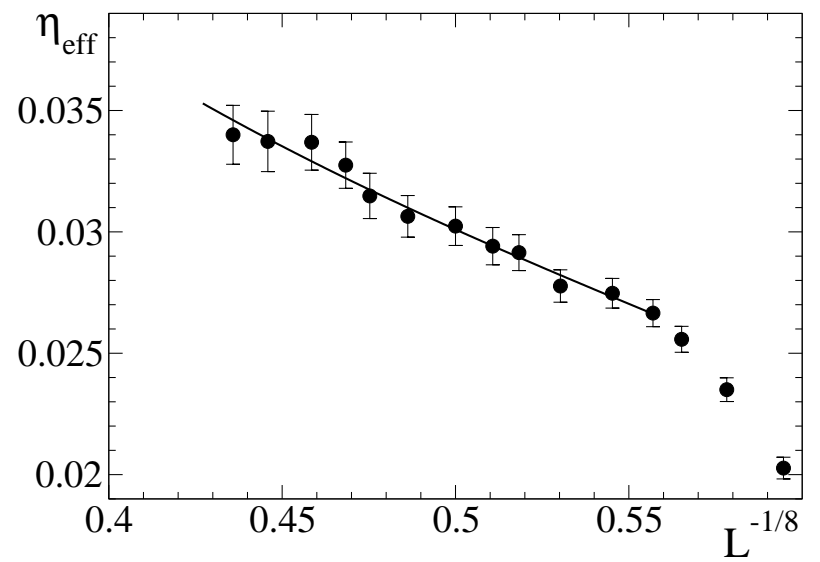

Fig. 6. The effective exponent $\eta_{\text {eff }}$ vs $L^{-1 / 8}$ at $\beta=\widetilde{\beta}_{c}(L)$. The solid line is the fit with $\eta=\omega=1 / 8$

mates at a larger $L_{\min }$, but have smaller statistical errors. Note that the decrease of the standard error for $\eta_{3}$ in Table 3 at $L_{\min }=64$ as compared to $L_{\min }=48,54$ is the effect of the already mentioned statistical correlations. These best $\eta$ values are very similar to the ones obtained before from the effective exponents.

Remarkable is the fact that a better estimation of the critical coupling, including two corrections to scaling instead of only one correction, leads to a worse agreement with the results of the perturbative RG theory. In particular, the value $\eta=0.0405(25)$ deviates above 0.036 (the upper limit of the best RG estimate $\eta=0.0335 \pm 0.0025$ of [33]) by $1.8 \sigma$. The observed here deviations suggest that $\eta$, probably, is larger than normally expected from the perturbative RG theory. The statistical errors, however, do not allow to make a strict conclusion.

\subsection{Fits with the exponents of the GFD theory}

As already discussed in Sec. 5, the pseudocritical coupling $\widetilde{\beta}_{c}(L)$ can be even better fit with the exponents $\nu=2 / 3$ and $\omega=1 / 8$ of the GFD theory than with those of the perturbative RG theory. We have also verified that the effective exponent $\eta_{\text {eff }}(L)$ is very well described by the ansatz (11) with $\eta=\omega=1 / 8$ (see Fig. 3) at a certain $\beta$ corresponding to a reasonable estimate of the critical coupling $\beta_{c}=0.221654604(18)$. At the pseudocritical coupling $\beta=\widetilde{\beta}_{c}(L)$, the effective exponent $\eta_{\text {eff }}(L)$ within $L \in[108,768]$ (extracted from the susceptibility data within $L \in[54,1536])$ can be well fit to (11) with fixed GFD exponents $\eta=\omega=1 / 8$, as shown in Fig. 6.

If the exponent $\eta$ is considered as a fit parameter at fixed $\omega=1 / 8$, then this fit yields $\eta=0.079(80)$ in agree- 
ment with the expected value $1 / 8$, although the statistical error is rather large in this case. Sufficiently accurate data for even larger $L$ values would be very helpful here to perform a more precise testing of the agreement.

We have verified that not only the effective exponent $\eta_{\text {eff }}$, but also the susceptibility data can be well described by the exponents $\eta=\omega=1 / 8$. In particular, $\chi^{2} /$ d.o.f of the fit with $\eta=\omega=1 / 8$ is 0.88 for the susceptibility data at $\beta=\widetilde{\beta}_{c}(L)$ within $L \in[64,1536]$. At $\beta=0.221654604 \simeq \beta_{c}$, the data can be well fit with $\chi^{2} /$ d.o.f $=1.01$ over a wider range of sizes $L \in[48,1536]$.

We have fitted also the $\partial Q / \partial \beta$ data to the finite-size scaling ansatz

$$
\frac{\partial Q}{\partial \beta} \simeq L^{1 / \nu}\left(A_{0}+A_{1} L^{-\omega}+A_{2} L^{-2 \omega}\right)
$$

These data can be well fit with the RG exponents, as well as with those of the GFD theory. We have compared the $\chi^{2} /$ d.o.f values of the fits within $L \in\left[L_{\min }, 1536\right]$ at $\beta=\widetilde{\beta}_{c}(L)$ in both cases. For three-parameter fits with fixed exponents $\nu=2 / 3$ and $\omega=1 / 8$ of the GFD theory, we have $\chi^{2} /$ d.o.f $=0.88,0.39$, and 0.43 at $L_{\min }=32,48$, and 64 , respectively. The corresponding values for $\nu=0.63$ and $\omega=0.8$ (the RG exponents) are $\chi^{2} /$ d.o.f $=0.56,0.39$, and 0.42 . Similarly, at a self-consistently estimated critical coupling $\beta=\beta_{c}$, we have $\chi^{2} /$ d.o.f $=0.93,0.47,0.50$ for the GFD exponents and $\chi^{2} /$ d.o.f $=0.63,0.47,0.48$ for the $\mathrm{RG}$ exponents at $L_{\text {min }}=32,48$, and 64 , respectively. The RG exponents provide somewhat better fits at $L_{\min }=32$, but the quality of fits is higher and practically equal for both sets of exponents at larger minimal sizes $L_{\text {min }}$.

In fact, reasonable fits with the exponents $\eta=1 / 8$ and $\nu=2 / 3$ are possible because of the value $1 / 8$ of the correction-to-scaling exponent $\omega$. A remarkably larger value, such as $\omega \simeq 0.8$, would make such fits not good. According to (11), the plot of the effective exponent $\eta_{\mathrm{eff}}$ vs $L^{-\omega}$ has to be almost linear at $L \rightarrow \infty$. If $\omega$ is as large as 0.8 , then $L^{-2 \omega}$ is already quite small for $L \geq 108$, so that a good linearity of the fit curve in Fig. 4 can be expected. However, the plot looks rather nonlinear. Moreover, it can be much better approximated by a straight line (within $L \in[108,768]$ ) in the $L^{-1 / 8}$ scale than in the $L^{-0.8}$ scale, as it is clearly seen comparing the plots in Figs. 4 and 6. According to this, it seems, in fact, very plausible that $\omega$ could be about $1 / 8$.

\section{Conclusions}

The analysis of the MC data for the $O(2)$ and $O(4)$ models below the critical point supports the recently published theoretical results [23], predicting that the Goldstone mode singularities in the $O(n)$ models are described by nontrivial exponents, as discussed in Sec. 2. Therefore, it has been important to verify the earlier predictions of this approach, called the GFD theory, concerning the critical point singularities. For this purpose, we have performed MC simulations of the 3D Ising model for very large lattices with linear size up to $L=1536$, using a parallel implementation of the Wolff single cluster algorithm. The finite-size scaling analysis shows that the actual data can be reasonably well interpreted with the usual critical exponents $\eta \simeq 0.0335, \nu \simeq 0.63$, and $\omega \simeq 0.8$ of the perturbative RG theory, and can also be well described by a different set of critical exponents, $\eta=\omega=1 / 8$ and $\nu=2 / 3$, found within the GFD theory. The validity of the fits with the latter set of exponents depends on whether the correction-to-scaling exponent $\omega$ can, indeed, be as small as $1 / 8$. We have demonstrated in Sec. 6.2 that this is a very plausible scenario.

We have performed a high accuracy estimation of the critical coupling $\beta_{c}$, combined with a sophisticated fitting of the data, including the leading, as well as subleading, corrections to scaling, to distinguish between the two sets of the critical exponents. We have found that a self-consistent estimation of $\beta_{c}$ and $\eta$, assuming the RG exponents $\nu \simeq 0.63$ and $\omega=0.8$, gives somewhat larger values of $\eta$ (e.g., $\eta=0.0397(28)$ and $\eta=0.0405(25)$ when two corrections to scaling are included) than the value $0.0335 \pm 0.0025$ expected from the most accurate resummation of the $\mathrm{RG}$ perturbative series [33]. These discrepancies are not large enough to make a strict conclusion about an inconsistency. On the other hand, the observed gradual increase in the effective exponent $\eta_{\text {eff }}(L)$ with growing system size $L$ is just expected if $\eta=\omega=1 / 8$ and $\nu=2 / 3$ are the correct asymptotic exponents. Therefore, it would be very interesting and important to see whether such an increase to even larger $\eta_{\text {eff }}$ values is supported by the data for even larger lattice sizes.

This work was made possible by the facilities of the Shared Hierarchical Academic Research Computing Network (SHARCNET:www.sharcnet.ca). It is based on the research presented at the 5th International Conference "Physics of Liquid Matter: Modern Problems" (Kyiv, Ukraine, 2010). R.M. acknowledges the support from the NSERC and CRC program. We thank also the DEISA Consortium (www.deisa.eu), funded through the EU FP7 project RI-222919, for support within the DEISA Extreme Computing Initiative. 
1. L. Onsager, Phys. Rev. 65, 117 (1944).

2. B. McCoy and T.T. Wu, The Two-Dimensional Ising Model (Harvard University Press, 1973).

3. R.J. Baxter, Exactly Solved Models in Statistical Mechanics (Academic Press, London, 1989).

4. P.D. Francesco, P. Mathieu, and D. Sénéchal, Conformal Field Theory (Springer, New York, 1997).

5. M.J. Huang and T.Y. Kang, Int. J. of Thermal Sci. 50 (7), 1156 (2011).

6. R.V.N. Melnik, X.L. Wei, and G. Moreno-Hagelsieb, J. of Biol. Systems 17 (3), 425 (2009).

7. T. Fiedler, I.V. Belova, and G.E. Murch, Computational Materials Science 47 (3), 826 (2010).

8. D. Zhang and R.V.N. Melnik, Applied Stochastic Models in Business and Industry 25 (5), 565 (2009).

9. J.K. Deng, X.D. Ding, T. Lookman, T. Suzuki, K. Otsuka, J. Sun, A. Saxena, and X.B. Ren, Phys. Rev. 81 (22), 220101 (2010).

10. M.E.J. Newman and G.T. Barkema, Monte Carlo Methods in Statistical Physics (Clarendon Press, Oxford, 1999).

11. T. Preis, P. Virnau, W. Paul, and J.J. Schneider, J. of Comput. Phys. 228 (12), 4468 (2009).

12. E. Martinez, P.R. Monasterio, and J. Marian, J. of Comput. Phys. 230 (4), 1359 (2011)

13. J.A. Redinz and A.C.N. de Magelhaes, Phys. Rev. B 51, 2930 (1995).

14. T. Hara and H. Tasaki, J. Stat. Phys. 47, 92 (1987).

15. C. Bagnuls and C. Bervillier, Phys. Rep. 348, 91 (2001).

16. J. Berges, N. Tetradis, and C. Wetterich, Phys. Rep. 363, 223 (2002).

17. J. Kaupužs, Int. J. Mod. Phys. B 23, 5935 (2009).

18. I.D. Lawrie, J. Phys. A: Math. Gen. 18, 1141 (1985).

19. U.C. Tuber and F. Schwabl, Phys. Rev. B 46, 3337 (1992).

20. L. Schäfer, H. Horner, Z. Phys. B 29, 251 (1978).

21. R. Anishetty, R. Basu, N.D. Hari Dass, and H.S. Sharatchandra, Int. J. Mod. Phys. A 14, 3467 (1999).

22. P. Hasenfratz and H. Leutwyler, Nucl. Phys. B 343, 241 (1990).

23. J. Kaupužs, Progress of Theoretical Physics 124, 613 (2010).

24. J. Kaupužs, Ann. Phys. (Leipzig) 10, 299 (2001).

25. J. Kaupužs, R.V.N. Melnik, and J. Rimšāns, Eur. Phys. J. B 55, 363 (2007)

26. J. Kaupužs, R.V.N. Melnik, and J. Rimšāns, Communications in Computational Physics 4, 124 (2008).

27. J. Kaupužs, R.V.N. Melnik, and J. Rimšāns, Phys. Lett. A 374, 1943 (2010).
28. D.J. Amit, Field Theory, the Renormalization Group, and Critical Phenomena (World Scientific, Singapore, 1984).

29. Shang-Keng Ma, Modern Theory of Critical Phenomena (Benjamin, New York, 1976).

30. J. Zinn-Justin, Quantum Field Theory and Critical Phenomena (Clarendon Press, Oxford, 1996).

31. H. Kleinert and V. Schulte-Frohlinde, Critical Properties of $\phi^{4}$ Theories (World Scientific, Singapore, 2001).

32. A. Pelissetto and E. Vicari, Phys. Rep. 368, 549 (2002).

33. R. Guid and J. Zinn-Justin, J. Phys. A 31, 8103 (1998).

34. M. Hasenbusch, Int. J. Mod. Phys. C 12, 911 (2001).

35. A.L. Tseskis, J. Exp. Theor. Phys. 75, 269 (1992).

36. Z-D. Zhang, Philosophical Magazine 87, 5309 (2007).

37. V.N. Bondarev, Phys. Rev. E, 77, 050103(R) (2008).

38. V.N. Bondarev, Eur. Phys. J. B, 77, 153 (2010).

39. J. Kaupužs, Int. J. Mod. Phys. C 16, 1121 (2005).

40. J. Kaupužs, Int. J. Mod. Phys. C 17, 1095 (2006).

41. J. Kaupužs, e-print cond-mat/0610015 (2010).

42. J. Kaupužs, J. Rimšāns, and R.V.N. Melnik, Phys. Rev. E 81, 026701 (2010).

43. L.N. Shur and P. Butera, Int. J. Mod. Phys. C 9, 607 (1998).

44. S. Mertens and H. Bauke, Phys. Rev. E 69, 055702(R) (2004)

45. Y. Deng and H.W.J. Blöte, Phys. Rev. E 68, 036125 (2003).

46. J.A. Plascak, A.M. Ferrenberg, and D.P. Landau, Phys. Rev. E 65, 066702 (2002).

Received 04.12.2010

КРИТИЧНІ ЯВИЩА І ФАЗОВІ ПЕРЕХОДИ

У ВЕЛИКИХ ГРАТКАХ У НЕПЕРТУРБАТИВНИХ

ПІДХОДАХ, ЩО ОСНОВАНІ НА МЕТОДІ МОНТЕ-КАРЛО

Дж. Каупуш, Дю. Рімшанс, Р.В.Н. Мелъник

$\mathrm{P}$ е $з$ ю м е

Розглянуто критичні явища і ефекти голдстоунівської моди у спінових моделях з $O(n)$ симетрією обертання. Починаючи із сингулярностей голдстоунівської моди у $X Y$ і $O(4)$ моделях, даємо короткий огляд різних теоретичних концепцій і сучасних результатів моделювання методом Монте-Карло. Вони відповідають недавнім результатам теорії групування діаграм Фейнмана (ГДФ) і показують, що ці сингулярності описуються певними нетривіальними експонентами, які відрізняються від передбачуваних раніше пертурбативними підходами. Наведено недавні результати Монте-Карло моделювання у тривимірній моделі Ізинга для граток з лінійними розмірами до $L=1536$, що набагато більше за $L \leq 128$ із зазвичай застосовними у 
скінченновимірному скейлінг-аналізі. Ці результати отримано з використанням паралельної ОреnMP реалізації однокластерного алгоритму Волфа. У припущенні загальноприйнятої експоненти $\omega \approx 0,8$ для скейлінгової поправки скінченновимірний скейлінг-аналіз критичної експоненти $\eta$ показує, що $\eta$ по- винна бути дещо більшою, ніж 0,0335 \pm 0,0025 за пертурбативною теорією ренормгрупи. Більше того, знайдено, що реальні дані можуть бути добре описані різними критичними експонентами $(\eta=\omega=1 / 8$ і $\nu=2 / 3)$, що отримані в теорії ГДФ. 